International Journal of Environment, Agriculture and Biotechnology
Vol-6, Issue-3; May-Jun, 2021
J Journal Home Page Available: https://ijeab.com/
Journal DOI: $10.22161 /$ ijeab

Article

Peer-Reviewed Journal

\title{
Analysis of the functioning of the Local Development Fund (FDL) of the Pokola Community Development Series
} (Congo)

\section{Analyse du fonctionnement du Fond de Développement Local (FDL) de la Série de Développement Communautaire de Pokola (Congo)}

\author{
Pierre Mbete ${ }^{1}$, Gilles Freddy Mialoundama Bakouetila ${ }^{2}$, Ayessa Leckoundzou ${ }^{1.4}$, Gildas \\ Ricklin Chablys Obimbola ${ }^{1}$, Rodolphe Aristide Goma ${ }^{3}$, Henri Boukoulou ${ }^{2}$
}

${ }^{1}$ Laboratoire d'Ecologie Appliquée et de l'Environnement, Ecole Nationale Supérieure d'Agronomie et de de Foresterie, Université Marien NGOUABI, Congo. Tél : (+242) 0666118 98, BP : 69, Brazzaville, Congo. Email : mbetedavid@gmail.com

${ }^{2}$ Laboratoire d'Economie et Sociologie Rurales, Ecole Nationale Supérieure d'Agronomie et de de Foresterie, Université Marien NGOUABI, Congo. Tél : (+242) 0660367 67, BP : 419, Brazzaville, Congo. Email : gmialoundama2016@gmail.com ${ }^{3}$ Centre National d'Inventaire et d'Aménagement des Ressources Forestières et Fauniques, Ministère de l'Economie Forestière, Congo. Tél : (+242) 0695013 71. Email : rodolphegoma@yahoo.fr

${ }^{4}$ Institut national de Recherche Forestière (IRF), Ministère de la Recherche Scientifique et de l'Innovation Technologique Tél : (+242) 055648805/ 066389495, BP : 419, Brazzaville, Congo. Email : ayessa03@ gmail.com

Auteur correspondant :ayessa03@gmail.com\&mbetedavid@gmail.com / (+242)06 6611898

Received: 01 Apr 2021; Received in revised form: 07 May 2021; Accepted: 20 May 2021; Available online: 08 Jun 2021 (C)2021 The Author(s). Published by Infogain Publication. This is an open access article under the CC BY license (https://creativecommons.org/licenses/by/4.0/).

Abstract - This study aims to assess the functioning of the Local Development Fund (FDL) of the UFA Pokola Community Development Series in Congo. It is based on the documentary review, semi-structured interviews with resource persons, household managers as well as the holding of focus groups. The results indicate that the local communities and indigenous populations (CLPA) are involved in the management bodies of the Community Development Series, bodies responsible for the management of the FDL. This fund is mainly supplied by royalties from the exploitation of forest species, it is intended to finance the micro-projects of the CLPAs. As a result, he is well known to the CLPAs of UFA Pokola (81\%). Between 2007 and 2016, the FDL mobilized 182,214,333 FCFA, including 145,274,472 FCFA, made it possible to finance nearly 69 micro-projects for the benefit of the CLPAs. Plant production remains the main sector where investments have been directed (64\%). There is also a spatial disparity in the investments of the FDL, the commune of Pokola benefited from 54\% of the funds invested. The natives have benefited little from the spin-offs of this mechanism for sharing the benefits of logging.

Keywords- Local development fund, local communities and indigenous populations, royalty, exploitation, Congo.

Résumé- La présente étude vise à évaluer le fonctionnement du Fonds de Développement Local (FDL) de la Série de Développement Communautaire de l'UFA Pokola au Congo. Elle se base sur la revue documentaire, des entretiens semi-structurés avec les personnes ressources, les responsables de ménages 
ainsi que la tenue des focus groups. Les résultats indiquent que les communautés locales et populations autochtones (CLPA) sont impliquées dans les organes de gestion de la Série de Développement Communautaire, organes responsables de la gestion du FDL. Ce fonds est essentiellement alimenté par les redevances de l'exploitation des essences forestières, il est destiné à financer les microprojets des CLPA. De ce fait, il est bien connu des CLPA de l'UFA Pokola (81\%). Entre 2007 et 2016, le FDL a mobilisé 182214333 FCFA dont 145274472 FCFA a permis de financer près de 69 microprojets aux profits des CLPA. La production végétale reste le principal secteur où les investissements ont été orientés (64\%). On note également une disparité spatiale des investissements du FDL, la commune de Pokola a bénéficié de 54 $\%$ des fonds investis. Les autochtones ont peu bénéficié des retombées de ce mécanisme de partage de bénéfice de l'exploitation forestière.

\section{Mots clés-Fonds de développement local, communautés locales et populations autochtones, redevance, exploitation, Congo.}

\section{INTRODUCTION}

Depuis la Conférence des Nations Unies sur l'Environnement et le Développement ayant abouti à un programme d'action pour le XXI ${ }^{\text {ème }}$ siècle (Ansallem, 2015) et le sommet de la terre tenu à Rio de Janeiro, en 1992, des nouveaux concepts de gestion durable et d'aménagement forestier durable sont apparus (FAO, 1996). Des politiques forestières sont formulées au niveau de chaque nation pour pérenniser et développer les fonctions économiques, écologiques et sociales des forêts ceci dans le cadre d'une gestion dite intégrée et participative qui assure de façon soutenue et durable la conservation et l'utilisation des ressources forestières et fauniques (Anonyme, 1999).

Dans le contexte de changement de paradigme dans l'aménagement forestier, la forêt se trouve au cœur des préoccupations environnementales et est aussi à la charnière de nouvelles attentes sociales (Boutefeu, 2005). Les modes de gestion appropriés seraient par conséquent ceux qui obéissent aux notions de démocratisation à la base, de libéralisation politique, de responsabilité collective (Nguinguiri, 1999); la gestion participative devient une nécessité.

La politique nationale en matière de gestion des ressources naturelles de nombreux pays est fortement influencée par les conventions internationales relatives à l'environnement et au développement durable qui sont ratifiées (Séne, 2014). Les projets d'aménagement forestier et de biodiversité comportent donc aujourd'hui une dimension sociale, question d'impliquer les populations dans la gestion durable des ressources naturelles (Robbin et al., 2006).

La forêt congolaise est productrice de valeur écologique, économique, sociale et joue un rôle essentiel dans la vie de l'économie nationale. Les activités de gestion forestière relèvent à la fois de l'administration publique (Etat, propriétaires des terres), du secteur privé ou parapublic (propriétaire des outils de production) et des communautés rurales qui possèdent des droits d'usages (N'zala, 2002). Le processus d'aménagement durable des forêts a démarré dans les années 2000 plus précisément dans le Secteur Forestier Nord (Nkeoua et al, 2005). Dans les concessions aménagées, on note un effort d'implication des communautés locales et populations autochtones, à travers des mécanismes appropriés, notamment la mise en place des conseils de concertation des Séries de Développement Communautaire (SDC), la création des Fonds de Développement Local, etc.

L'Unité Forestière d'Aménagement de Pokola est une concession forestière concédée à la société forestière Congolaise Industrielle des Bois en sigle CIB (Anonyme, 2007), du groupe international Olam. Le modèle de gestion de la société CIB-OLAM, entreprise pionnière en matière d'aménagement forestier au Congo, et de certification de gestion durable peut être pilote pour comprendre la problématique du Fond de Développement Local (FDL). Il avait été institué en 2007 un fonds de développement local d'une valeur $200 \mathrm{~F} \mathrm{CFA} / \mathrm{m}^{3}$ indexée à la production de bois commercialisable réalisée par l'exploitant forestier au cours d'une année civile. Ce fonds est géré par un conseil de concertation de la SDC, comprenant différentes parties prenantes, et date depuis plus de dix ans. Une décennie après la mise en cuvre du FDL de la Série de Développement Communautaire de l'Unité Forestière d'Aménagement (UFA) Pokola, son fonctionnement et son bilan restent encore mal connu et peu d'informations scientifiques sont disponibles. C'est pourquoi, la présente étude vise à évaluer le fonctionnement de ce FDL. De manière spécifique, il s'agit : de décrire l'organisation et le fonctionnement $\mathrm{du}$ FDL, analyser les ressources financières mobilisé et investit par ce fonds au profit des CLPA. 


\section{METHODOLOGIE}

\section{Localisation de la zone d'étude}

L'étude a été réalisée dans la Série de Développement Communautaire de l'Unité Forestière d'Aménagement (UFA) de Pokola, une entité située dans le département administratif de la Sangha au nord de la République du Congo(Figure 1). La concession forestière attribuée à la société CIB-Olamen 2002 possède une superficie de 452200 ha.L'UFA Pokolaest repartie en cinq (5) séries d'aménagement: production, conservation, protection, développement communautaire, recherche. Les Communautés Locales et Populations Autochtones vivent et réalisent les activités socioéconomiques dans la Série de
Développement Communautaire. La SDC est un espace affecté aux activités villageoises et au développement local, il est composé d'un ensemble de terroirs et finages villageois comprenant les forêts naturelles et artificielles, des terres agricoles, les jachères, les zones de pêche et de chasse. L'objectif de la SDC est de satisfaire les besoins des populations forestières en produits forestiers et d'améliorer leur revenu.

La SDC de l'UFA Pokola comprend une surface de 26490 hectares pour une population estimée à 15582 habitants. Elle compte 12 villages et une commune de plein exercice au sein de laquelle on trouve la scierie et les bâtiments administratifs de la société forestière CIB-Olam.

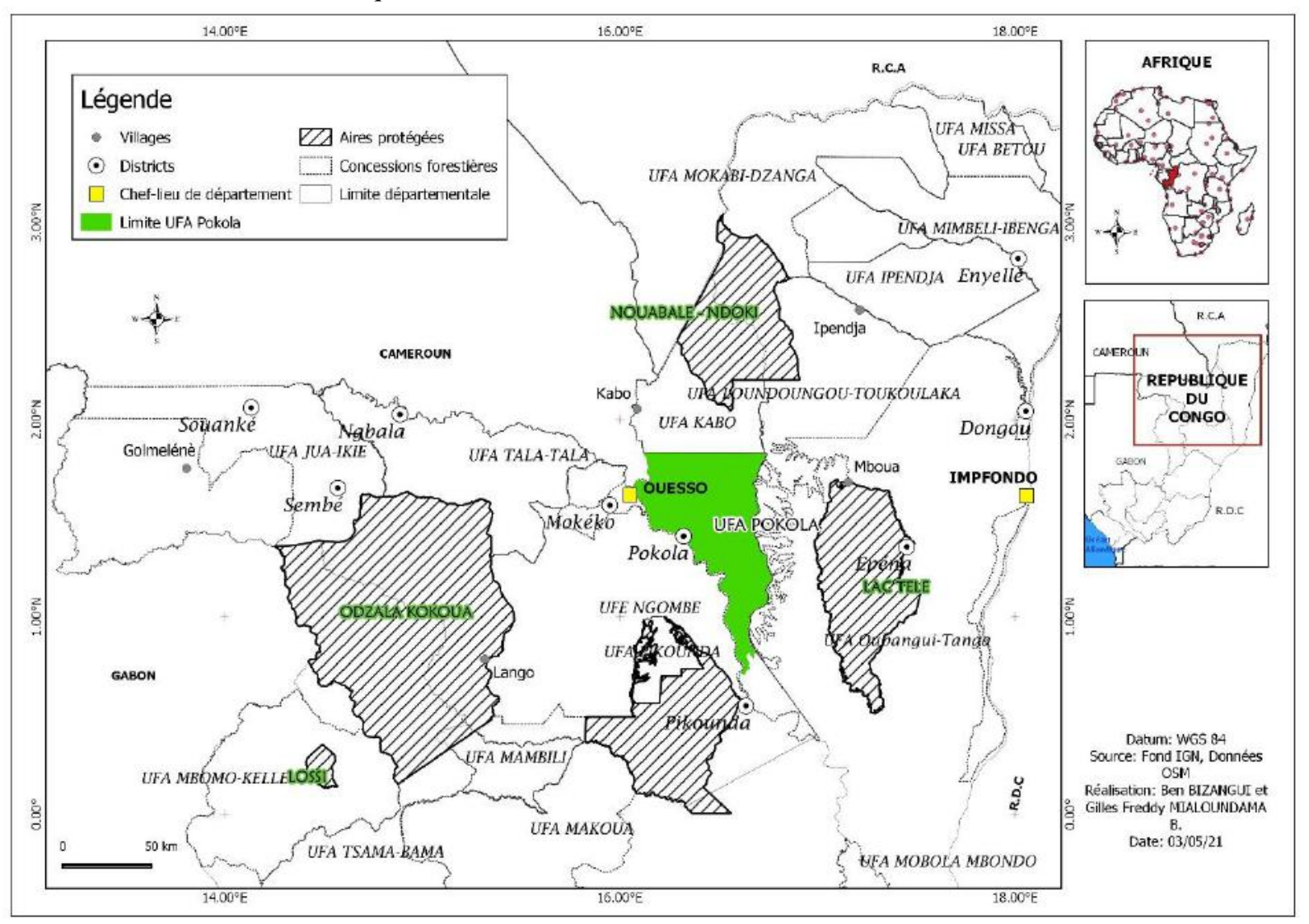

Fig.1 : Localisation de l'UFA Pokoladans le secteur forestier nord Congo

\section{Collecte des données}

La revue de la littérature a porté sur la documentation en lien avec l'objet de l'étude, notamment :

- les données juridiques et officielles sur l'exploitation forestière et la décentralisation au Congo (lois, décrets et arrêtés, économie forestière, etc.) ;

- les documents de la CIB (plan d'aménagement, rapport d'études et de missions, etc.
- les documents de gestion de la Série de Développement Communautaire et le conseil de concertation (plan de gestion de la SDC, procédures de gestion des fonds, règlement intérieur, compte rendu des sessions, preuves de calcul de la redevance et alimentation du compte du FDL, etc.) ;

- les autres études portant sur la gestion participative et l'implication des communautés locales et populations autochtones (CLPA) dans la gestion des ressources naturelles. 
L'exploitation de ces documents a permis une meilleure connaissance de la zone d'étude, une compréhension du cadre d'intervention du FDL dans la SDC ainsi que son bilan.

La revue de la littérature a été complétée par des entretiens individuels avec les personnes ressources ayant une connaissance du FDL et/ou ayant été impliquées dans le dispositif de sa mise en œuvre (agents de la cellule d'aménagement de la CIB-Olam, Organisation de la Société Civile, membres du conseil de concertation, responsables de la préfecture de la Sangha, maire de la commune de Pokola, chef de brigade de l'économie forestière ainsi que son Directeur départemental, chef de secteur agricole ainsi que son Directeur départemental, etc.). Ces entretiens semi-structurés ont été menés à l'aide des guides d'entretiens. Les données portaient sur le fonctionnement du FDL, son bilan et les contraintes de développement. La période concernée par la présente étude est celle allant de 2007 à 2016.

Des focus group ont été également tenus dans 8 villages sur les 12 que compte l'UFA Pokola. Ils se tenaient en présence du représentant du programme social de la CIB. Ils ont permis de recueillir des opinions relatives au fonctionnement du FDL, le financement des microprojets, les forces et les faiblesses de l'intervention du FDL. Des observations directes de quelques réalisations du FDL ont été également effectuées. Pour évaluer le niveau de connaissance des CLPA sur le FDL de Pokola, 331 personnes ont été interrogées dont 169 communautés locales (bantous) et 162 autochtones.

\section{Analyse des données}

La saisie et l'analyse des données quantitatives ont été réalisées au moyen du logiciel Excel 2013. Cependant, les données qualitatives issues des entretiens et de la revue documentaire ont fait l'objet d'une analyse decontenu. Cette analyse a consisté en un examen systématique et méthodique des documents textuels afin de décortiquer le texte en énoncé plus court et significatif pour en faire la synthèse des idées principales et secondaires.

\section{RESULTATS}

\subsection{Les parties prenantes de la SDC de l'UFA Pokola}

D'après les dispositions de l'arrêté $\mathrm{n}^{\circ} 2668 /$ MDDEFE/CAB du 15 avril 2010, portant institution, organisation et fonctionnement du conseil de concertation de la SDC de l'UFA Pokola, les différents organes de gestion de la SDC sont : (i) le conseil de concertation, (ii) la coordination technique, (iii) le comité de suivi et d'évaluation, (iv) le bureau exécutif, (v) le comité d'achat. Le tableau 1 présente les différentes parties prenantes des trois premiers organes de gestion

Tableau 1 : Organisation du conseil de concertation, la coordination technique, le comité d'évaluation de la SDC de l'UFA Pokola

\begin{tabular}{|c|c|c|c|}
\hline & Conseil de concertation & Coordination technique & $\begin{array}{l}\text { Comité de suivi et } \\
\text { d'évaluation }\end{array}$ \\
\hline \multirow{4}{*}{ 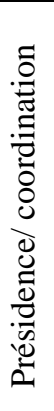 } & Président : représentant du conseil départemental & $\begin{array}{l}\text { Chef de brigade de } \\
\text { l'économie forestière }\end{array}$ & $\begin{array}{l}\text { Président : représentant de } \\
\text { la préfecture }\end{array}$ \\
\hline & $\begin{array}{l}\text { Premier vice-président : représentant des communautés } \\
\text { villageoises }\end{array}$ & & $\begin{array}{l}\text { Vice-président : } \\
\text { représentant DDEF }\end{array}$ \\
\hline & $\begin{array}{l}\text { Deuxième vice-président : représentant de la société } \\
\text { forestière }\end{array}$ & & \\
\hline & $\begin{array}{l}\text { Rapporteur : chef de brigade de l'économie forestière, } \\
\text { coordonnateur technique de la SDC }\end{array}$ & & \\
\hline \multirow{5}{*}{ 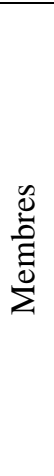 } & Représentant de la préfecture & Chef de secteur agricole & Représentant de la CIB \\
\hline & Sous-préfet du district de Pokola & Chef de poste agricole & $\begin{array}{l}\text { Représentant des ONG } \\
\text { locales - }\end{array}$ \\
\hline & Directeur départemental de l'économie forestière & $\begin{array}{l}\text { Représentant des ONG } \\
\text { œuvrant dans l'UFA }\end{array}$ & \\
\hline & $\begin{array}{l}\text { Directeur départemental de l'aménagement du } \\
\text { territoire }\end{array}$ & $\begin{array}{l}\text { Représentants des } \\
\text { communautés de village }\end{array}$ & \\
\hline & Directeur départemental de l'environnement & $\begin{array}{l}\text { Représentant de la société } \\
\text { forestière }\end{array}$ & \\
\hline
\end{tabular}




\begin{tabular}{|l|l|l|l|}
\hline & Directeur départemental de l'agriculture & Personne ressource & \\
\hline & Directeur départemental de l'élevage & & \\
\hline & Directeur départemental de la pêche & & \\
\hline & Deux représentants de la société forestière CIB & & \\
\hline $\begin{array}{l}\text { Des représentants des communautés des villages, dont } \\
\text { certains semi-nomades et femmes }\end{array}$ & & \\
\hline $\begin{array}{l}\text { Des représentants d'ONG en activité dans l'unité } \\
\text { forestière }\end{array}$ & & \\
\hline & Toute personne appelée pour sa compétence & & \\
\hline
\end{tabular}

Le bureau exécutif est composé d'un président (Représentant du Conseil départemental de la Sangha) et de deux membres, le chef de brigade de la Direction Départemental de l'Economie Forestière ainsi qu'un représentant de la société forestière. Cependant le comité d'achat est présidé par un représentant des ONG, assisté de 4 membres (un représentant de la société forestière, 3 personnes des villages bénéficiaires).

La gestion de la série de développement communautaire incombe au conseil de concertation impliquant tous les acteurs communautaires et présidé par un représentant du conseil départemental. Ce conseil de concertation a pour objectifs :(i) adopter le plan de gestion de la SDC de l'UFA, (ii) examiner et approuver les microprojets et activités prévues dans la SDC, (iii) examiner et approuver le budget du fonds de développement local, (iv) approuver les critères d'éligibilité des microprojets, (v) adopter le plan de gestion de la SDC et les critères d'éligibilité des microprojets, (vi) examiner et faciliter le règlement des différends entre les parties prenantes impliquées dans la gestion des ressources naturelles et le développement socio-économique de la SDC de l'UFA, (vii) examiner et adopter le programme et les rapports d'activités de la coordination technique du Conseil de concertation.

La participation des CLPA aux décisions de la gestion forestière est plus effective avec le fonctionnement du conseil de concertation et ses organes. Le conseil de concertation est une forme de démocratie participative au niveau locale, c'est une plateforme de concertation multiacteurs.

La coordination technique est le second organe de gestion de la SDC, elle est chargée de : (i) suivre la réalisation des microprojets, (ii) assister les populations dans la réalisation de leurs activités, (iii) préparer les documents à soumettre au conseil de concertation, (iv) suivre la mise en œuvre du plan de gestion de la SDC de l'UFA Pokola, (v) informer et sensibiliser les populations sur la mise en œuvre du plan d'aménagement et du plan de gestion de la SDC de l'UFA Pokola, (vi) mettre en place et gérer la base de données.
Le comité de suivi et évaluation est l'organe de gestion de la SDC de l'UFA Pokola, chargé d'assurer le suivi des activités menées dans la SDC et de procéder à une évaluation technique et financière des activités menées.

Dans le but de lutter contre la pauvreté et de contribuer à l'amélioration des conditions de vies des populations, un Fonds de Développement Local (FDL) est créé. Il est alimenté par une redevance de 200 FCFA par $\mathrm{m}^{3}$ indexée sur le volume de bois commercialisable exploité dans l'UFA, la subvention du Conseil Départementale de la Sangha, les dons et legs. Le FDL est destiné à :

- financer les microprojets d'intérêt communautaire dans les limites de la SDC à hauteur de $85 \%$ des recettes annuelles ;

- assurer le fonctionnement du Conseil de Concertation et des organes techniques qui composent à hauteur de $15 \%$ des recettes annuelles.

\subsection{Niveau de connaissance du FDL par les Communautés Locales et Populations Autochtones}

Les Communautés Locales et Populations Autochtones (CLPA) de la Série de Développement Communautaire de l'UFA Pokola ont une connaissance du Fonds de Développement Local (FDL). La proportion de $91 \%$ des populations locales bantous connait le FDL, contre $9 \%$ qui affirment ne pas connaitre ce mécanisme de partage de bénéfice de l'exploitation forestière. Cela témoigne d'une bonne sensibilisation faite pour vulgariser cet outil de financement des microprojets des CLPA.

Le FDL est globalement bien connu des populations autochtones $(70 \%)$. Toutefois $30 \%$ des autochtones interrogés ont aucune connaissance de ce mécanisme de financement des microprojets. Le faible niveau de connaissance du FDL (30\%)estmarqué chez les autochtones, soit 36 femmes parmi les 48 autochtones n'ayant pas connaissance de ce fonds (tableau 2). 
Tableau 2 : Evaluation du niveau de connaissance des CLPA sur le FDL

\begin{tabular}{|l|c|c|c|c|}
\hline \multirow{2}{*}{ Genre } & \multicolumn{2}{|c|}{$\begin{array}{c}\text { Connaissance du FDL par la } \\
\text { population locale (Bantou) }\end{array}$} & \multicolumn{2}{c|}{$\begin{array}{c}\text { Connaissance du FDL par les } \\
\text { autochtones }\end{array}$} \\
\cline { 2 - 5 } & Oui & Non & Oui & 12 \\
\hline Homme & 101 & 9 & 67 & 36 \\
\hline Femme & 53 & 6 & 47 & $\mathbf{4 8}$ \\
\hline Total & $\mathbf{1 5 4}$ & $\mathbf{1 5}$ & $\mathbf{1 1 4}$ & Non \\
\hline
\end{tabular}

\subsection{Evaluation des ressources financières mobilisées par le FDL}

Pendant la période décennale (2007-2016), le FDL a été alimenté par les redevances payées par la société forestière CIB-Olam, la redevance de l'exploitation du bois de valeur marchande dans la zone de développement communautaire de Pokola de la SDC, les contributions du conseil départemental de la Sangha et de l'Organisation Internationale des Bois Tropicaux (OIBT) à travers le Projet de Gestion des Ecosystèmes Périphériques au Parc (PROGEPP). Au cours de cette période un montant de 182.214.333 FCFA a été mobilisé. La proportion de $85 \%$ de ce montant était orientée vers l'investissement à travers le financement des microprojets, soit 154.882.183 FCFA. Le reste $(15 \%)$ était consacré au fonctionnement des organes de gestion de la Série de Développement Communautaire, soit 27.332.150 FCFA.

L'exploitation du bois dans les Unités Forestières de Production (UFP) 1 et $2\left(618.632 .724 \mathrm{~m}^{3}\right.$ de volume de bois roulés) a généré au FDL un montant de 123.726.543 FCFA.L'exploitation du bois de valeur marchande dans la SDC a été formalisée en 2013 à travers un protocole d'accord signé entre la société forestière CIB-Olam et le conseil départemental de la Sangha. La récupération de bois de valeur économique par la CIB-Olam dans la zone de développement communautaire de Pokola a eu lieu en 2014 et a généré une somme de 53.256.625 FCFA. La redevance forestière payée varie entre 1000 FCFA et 2000 FCFA par essence forestière exploitée. La base de taxation des essences forestières de haute valeur varie entre 1500 et 2000 FCFA $/ \mathrm{m}^{3}$, c'est le cas du Doussié (Afzeliabipindensis) taxé à $2000 \mathrm{FCFA} / \mathrm{m}^{3}$, du Sapelli (Entandrophragmacylindricum) et du Sipo (Entandrophragma utile) taxés à $1500 \mathrm{FCFA} / \mathrm{m}^{3}$. Les autres essences forestières sont taxées à $1000 \mathrm{FCFA} / \mathrm{m}^{3}$, c'est le cas du Wengué (Milletialaurentii), du Tali (Erythrophleumivorense), $\mathrm{du}$ Padouk (Pterocarpussoyauxii), etc.

La redevance issue de l'exploitation du bois dans l'UFP 1 et 2 ainsi que dans la SDC par la CIB-Olam ont contribué à près de $97 \%$ des fonds mobilisés par le FDL au cours de la période 2007-2016. La CIB-Olam est donc le principal pourvoyeur des ressources financières du FDL. Cependant les apports de l'OIBT à travers le PROGEPP et du conseil départemental de la Sangha restent très marginal, soit respectivement 3.231.165 FCFA et 2.000.000 FCFA (figure 2).

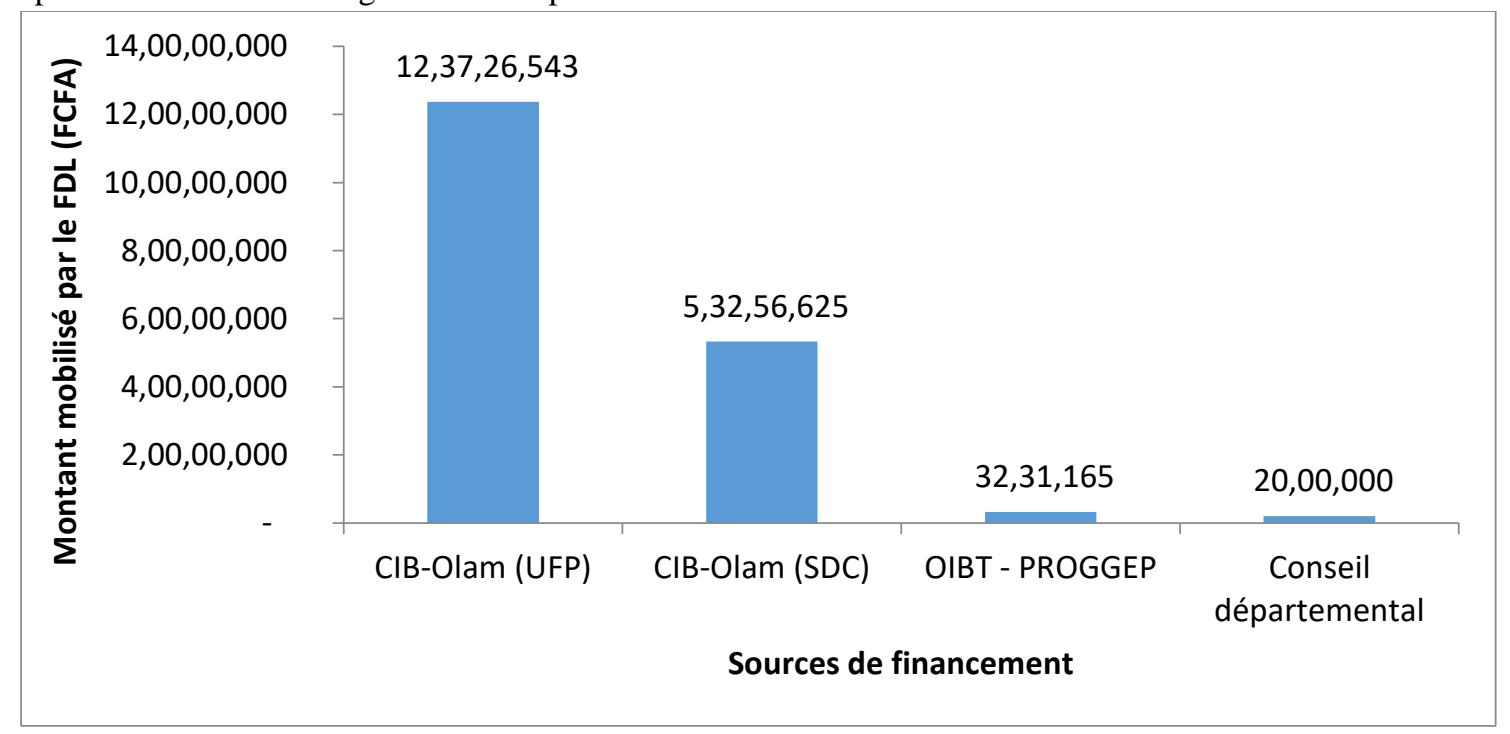

Fig.2: Répartition des sources de financement mobilisées par le FDL de l'UFA Pokola 
3.4 Analyse des investissements du FDL de l'UFA Pokola

\subsubsection{Répartition des investissements du FDL par secteur d'activité}

Dans la période de 2008 à 2016, le FDL a financé 69 microprojets aux bénéfices des communautés locales et populations autochtones, soit un montant investit de 145.274.472 FCFA. Les investissements dans les microprojets de production végétale (45 projets) représentent 93.577.872 FCFA, soit $64 \%$ de l'ensemble des investissements. Dans ce secteur, les microprojets de la culture de cacaoyer (Theobroma cacao) et du manioc (Manihotesculenta) sont les plus financés, soit

Tableau 3 : Secteur d'investissements du FDL et coût de réalisation des microprojets

\begin{tabular}{|l|c|c|c|}
\hline \multicolumn{1}{|c|}{$\begin{array}{c}\text { Secteur } \\
\text { d'investissement }\end{array}$} & Montant en FCFA & Nombre de microprojet & $\begin{array}{c}\text { Pourcentage des } \\
\text { investissements }(\%)\end{array}$ \\
\hline Production végétale & 93577872 & 45 & 64 \\
\hline Elevage & 14436600 & 8 & 4 \\
\hline Pêche & 6180000 & 9 & 20 \\
\hline Transport & 28580000 & 1 & 1 \\
\hline Habitat & 1500000 & 1 & 100 \\
\hline Pisciculture & 1000000 & $\mathbf{6 9}$ & $\mathbf{1 0 0}$ \\
\hline Total & $\mathbf{1 4 5 2 7 4 \mathbf { 4 7 2 }}$ & 5 & 1 \\
\hline
\end{tabular}

\subsubsection{Répartition inégale des investissements du FDL par localité de la SDC de Pokola}

Le FDLa réalisé des investissements dans toutes les localités de la Série de Développement Communautaire (SDC) de l'Unité Forestière d'Aménagement de Pokola (UFA). La commune de Pokola a bénéficié pratiquement de la moitié des fonds investit à l'échelle de l'UFA Pokola, soit $54 \%$ (tableau 4). On note une forte disparité entre les financements accordés par le FDL à la commune de Pokola et les villages de la SDC de l'UFA Pokola. Cette pratique prouve le dynamisme économique de la commune de Pokola, où le FDL est influencé par les acteurs clefs des organes de gestion de la SDC. Ces inégalités spatiales dans les investissements du FDL en faveur de la commune de Pokola semblent être en déphasage avec les recommandations des études socioéconomiques de bases ayant servi à l'élaboration du plan d'aménagement de l'UFA. Ces études ont fortement recommandé respectivement 55.709.350 FCFA et 33.868.522 FCFA. Le secteur de transport (transport par pirogue motorisée) représente le second secteur d'investissement de ces fonds, mobilisant $20 \%$ des investissements (28.580.000 FCFA). Près de 9 microprojets ont été financés dans ce secteur.Les investissements dans le secteur de l'élevage (aviculture, production ovine et caprine, production porcine) représentent $10 \%$ de l'ensemble des investissements, soit 14.436.600 FCFA pour 8 microprojets (tableau 3). Les investissements dans les autres secteurs d'activité sont faibles.

Tableau 4 : Répartition des investissements du FDL par localité de l'UFA Pokola

\begin{tabular}{|l|l|c|c|c|}
\hline \multicolumn{1}{|c|}{ Localité } & \multicolumn{1}{|c|}{ Statut } & Superficie (ha) & $\begin{array}{c}\text { Investissement du } \\
\text { FDL (FCFA) }\end{array}$ & $\begin{array}{c}\text { Part des } \\
\text { investissements (\%) }\end{array}$ \\
\hline Pokola & Commune & 15810 & 78448215 & 54 \\
\hline Ngangassa & Village & 365 & 8716468 & 6 \\
\hline
\end{tabular}




\begin{tabular}{|l|l|c|c|c|}
\hline Ikélemba & Village & 1640 & 8716468 & 6 \\
\hline Ngatongo & Village & 1410 & 8716468 & 6 \\
\hline Ngandzikolo & Village & 895 & 8716468 & 5 \\
\hline Mbirou & Village & 1705 & 7263724 & 4 \\
\hline Djaka & Village & 1085 & 5810979 & 3 \\
\hline Konda & Village & 385 & 5810979 & 3 \\
\hline Matali & Village & 1705 & 4358234 & 3 \\
\hline Bossendé & Campement & - & - & 4358234 \\
\hline Matoto & Village & 490 & & 6 \\
\hline
\end{tabular}

\subsubsection{Répartition des investissements du FDL entre les communautés locales Vs populations autochtones}

L'approche de financement des microprojets FDL dans les villages ne permet pas de faire une comparaison aisée entre les communautés Bantou et populations autochtones. Le village étant mixte, généralement un seul projet est financé pour ces deux communautés. La mise en œuvre semble être difficile et la répartition des bénéfices pas assez aisée du fait des relations de domination des bantous sur les populations autochtones. Les autochtones affirment n'être pas bénéficiaires des retombées des financements.

Dans les villages de Molembé,Ngangassa, Ngatongo, Djaka et Ikélemba par exemple, les autochtones sont parfois assistés en cas de maladies et lors des funérailles par les bénéfices issus de l'exploitation des pirogues motorisées. Si l'analyse comparative des investissements

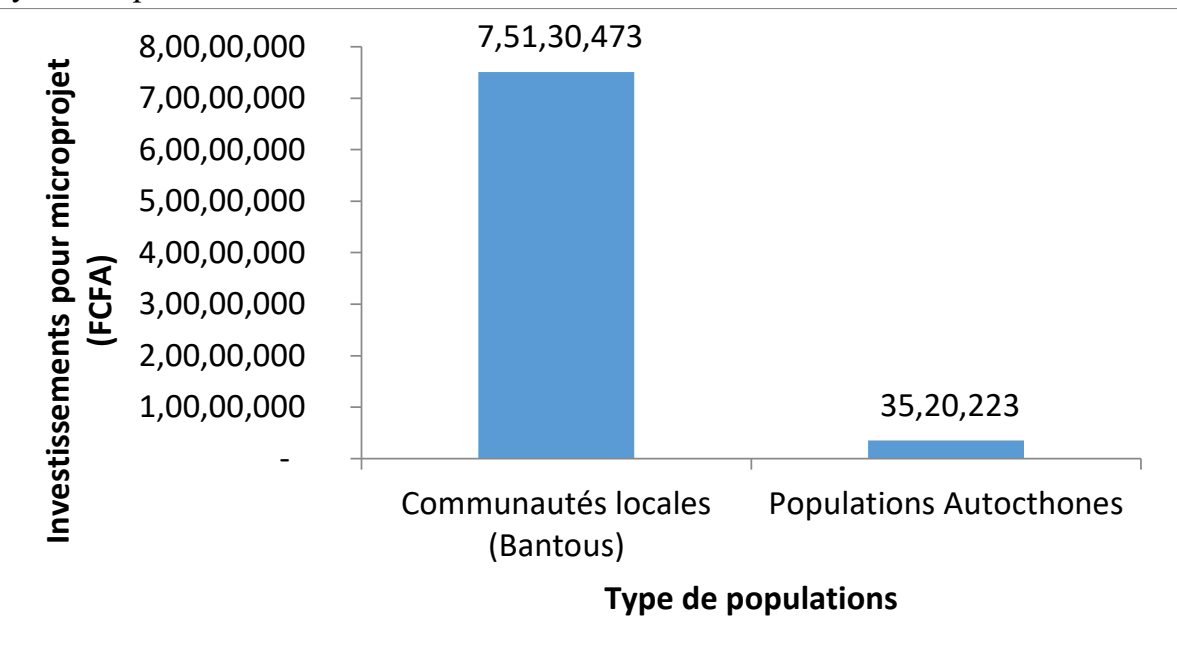

Fig.3: Répartition des investissements du FDL entre organisations bantous et autochtones

\subsubsection{Analyse des impacts du FDL}

Les investissements réalisés par le FDL visent l'autonomisation des communautés et ainsi contribuer à l'amélioration des conditions de vie des populations. Les microprojets dans le secteur du transport (pirogue entre bantou et autochtones est difficile à établir dans les villages, cela est plus aisé à réaliser à Pokola, car l'approche de financement a été séparée. Le FDL a privilégié les individualités, les groupements et associations. L'analyse de la figure 3 montre une faible prise en compte des préoccupations des groupes sociaux défavorisés (autochtones) par le dispositif FDL (figure 3). L'argumentaire avancé par certains gestionnaires n'est pas soutenable, semble-t-il que les populations autochtones n'adressent pas leur demande au FDL. Cette analyse devrait interpeller les décideurs du FDL, en vue de se conformer aux engagements de la CIB prévus dans les documents d'orientations (plan d'aménagement, mesures sociales et de gestion). 
la circulation des personnes et des biens le long de la rivière Sangha. Les bénéfices générés par la gestion des pirogues ont contribué à la construction d'une école, la prise en charges des frais scolaires et autres produits de premières importances, etc.

L'agriculture et la pêche sont les secteurs d'activités qui ont un impact significatif pour des raisons suivantes: contribuent à la sécurité alimentaire des villages, possibilité d'acquisition des intrants et de production. Cependant les microprojets dans les secteurs de l' 'élevage, l'habitat, et la pisciculture sont considérés comme des investissements à impacts limités du fait de leurs faibles apports dans les ménages.
L'initiative du FDL reste une bonne approche pour l'implication et la responsabilisation des CLPA, dans la gestion forestière. Il constitue une opportunité pour encourager la diversification de l'économie locale en s'appuyant sur des projets communautaires, gage du développement socioéconomique de la SDC. Toutefois les effets du financement de ces microprojets restent parfois mitigés et quasiment nul pour les populations autochtones. $\mathrm{Au}$ stade actuel, aucun microprojet n'a la capacité de s'autofinancer et se pérenniser à la fin de la subvention accordée. Après 10 ans de mise en œuvre, les microprojets FDL ont de la peine à assurer leurs visibilités.

Tableau 5:Impact des types d'investissementdu FDL sur les conditions de vie des CLPA

\begin{tabular}{|l|c|c|c|c|}
\hline \multirow{2}{*}{ Secteur d'activité } & \multicolumn{4}{|c|}{ Appréciations des impacts sur l'amélioration des vies des CLPA } \\
\cline { 2 - 5 } & $*$ impact limité & $\begin{array}{c}* * \text { impact } \\
\text { significatif }\end{array}$ & $\begin{array}{c}* * * \text { impact } \\
\text { important }\end{array}$ & $\begin{array}{c}\text { **** impact très } \\
\text { important }\end{array}$ \\
\hline Agricole & $*$ & $* *$ & & \\
\hline Elevage & $*$ & & & \\
\hline Pêche & & $* *$ & & \\
\hline Transport & $*$ & & & \\
\hline Habitat & $*$ & & & \\
\hline Pisciculture & & & & \\
\hline
\end{tabular}

Le FDL représente un modèle innovant de mécanisme de développement local, intégrant les différents niveaux de planification et d'exécution, de la communauté locale villageoise aux autorités départementales. C'est un outil financier pour le partage de bénéfices de la rente forestière. A travers le financement des microprojets, le FDL a contribué efficacement à faire asseoir la paix sociale entre la société forestière CIB-Olam et les CLPA de l'UFA Pokola. On assiste également à une nette prise de conscience, par les populations à la base, de leur nouveau rôle dans la gestion forestière et cela est vécu comme un impact positif à travers le dialogue permanent institué entre les acteurs parties prenantes à la gestion forestière. Le conseil de concertation apparait aujourd'hui comme un organe régulateur permettant de concilier les logiques des différents acteurs (Etat, exploitant forestier, ONG et CLPA) dans la gestion des ressources forestières dans l’UFA Pokola.

Les microprojets financés par le FDL ont également entrainé des faibles impacts négatifs. Ils ont été à l'origine de l'émergence de petits conflits sociaux entre les bénéficiaires et non bénéficiaires du financement des microprojets ou du partage des avantages socioéconomiques obtenus. Ces conflits peuvent être atténués par les actions d'accompagnement des acteurs dans la gestion des microprojets.

La réalisation des microprojets dans le secteur de la production végétale (cacaoyer, manioc, etc.) a entrainé la perte de la couverture végétale, de la biodiversité et l'émission des gaz à effet de serre. La pratique d'agriculture itinérante sur brûlis a contribué à la déforestation, le taux de déforestation sur la période de 2007 à 2016 a été évalué à $0,96 \%$ de la superficie de la SDC. Une superficie de 106 ha a été défrichée dont 56 ha pour les cultures vivrières et 50 ha pour la culture du cacaoyer.

\section{DISCUSSION}

La fiscalité forestière décentralisée contribue à améliorer la participation et l'implication des populations locales (Fomété, 2001). Le Fonds de Développement Local (FDL) est un instrument de gestion participative des ressources naturelles, qui permet d'impliquer les Communautés Locales et Populations Autochtones (CLPA) dans la gestion durable d'une concession forestière aménagée. La 
présente étude a montré que le FDL contribue au développement socioéconomique local en finançant les microprojets des CLPA vivant dans la Série de Développement Communautaire (SDC) de l'Unité Forestière d'Aménagement (UFA) Pokola. Ce fonds est donc un modèle innovant de mécanisme de développement local, intégrant dans les organes de gestion du FDL (conseil de concertation et la coordination technique)les CLPA ainsi que les autorités du conseil départemental de la Sangha.

Le FDL de la SDC de l'UFA Pokola est alimenté principalement $(97 \%)$ par les redevances payéespar la société forestière CIB-Olam à la suite de l'exploitation des unités forestières de production (UFP) et des essences forestières dans la SDC de l'UFA Pokola. Ce fonds permet aux CLPA de bénéficier tant soit peu des retombées de l'aménagement forestier. Dans le secteur forestier Camerounais, deux types de taxes décentralisées contribuent au développement local, notamment la redevance forestière annuelle (RFA) et la taxe dite de 1000 FCFA (Fometé, 2001). Ce même auteur, affirme que la redevance forestière annuelle est un outil de fiscalité décentralisé où $50 \%$ du montant est destiné aux communes dont $10 \%$ versés aux communautés riveraines. Cependant, la taxe dite des 1000 FCFA (1000 FCFA/m³ de bois exploités) ne s'applique qu'aux titres d'exploitation de vente et de coupe de bois, elle est destinée à des projets locaux d'œuvres sociales (écoles, routes, etc.).

Les résultats de l'étude montrent que le financement des microprojets par le FDL dans l'ensemble des localités de la SDC de Pokola a permis d'atténuer les revendications vis-à-vis de la société forestière et instaurer la paix sociale. L'implication des CLPA dans les organes de gestion du FDL permet d'augmenter leur participation dans la gestion de l'UFA Pokola. La participation ou l'implication des communautés dans la prise de décision de manière formelle et informelle est l'un des principes de bonne gouvernance des forêts (FapaNanfack et al., 2020). L'approche participative est un instrument innovant de gouvernance forestière qui prend en compte toutes les parties prenantes, notamment les populations locales (Koffi Kouamekan, 2013). Elle permet aux populations concernées par l'exploitation du domaine forestier d'en être les acteurs, au même titre que l'Etat et les exploitants forestiers (Collas de Chatelperron, 2005). Dans ce contexte, le FDL alimenté principalement par les redevances de l'exploitation des essences forestières dans les Unités forestière de Production (200 FCFA $\left./ \mathrm{m}^{3}\right)$ et dans la SDC (1000 à $\left.2000 \mathrm{FCFA} / \mathrm{m}^{3}\right)$, est donc une véritable opportunité pour les CLPA de bénéficier des retombées de l'exploitation forestière et de susciter leur participation dans les organes de gestion de la SDC; organes comprenant différentes parties prenantes.

En République du Congo, 1'effort d'implication des CLPA dans la gouvernance forestière est surtout constaté dans les concessions forestières aménagées. C'est le cas de l'UFA Ngombé où les CLPA sont impliqués dans la gestion du FDL à travers le conseil de concertation (MankeriNdobolo, 2020). Cependant dans les concessions forestières non aménagées dont la plupart se situe dans les secteurs forestiers centre et sud, la gestion participative est loin d'être une réalité. Par conséquent, les CLPA ne sont pas impliquées dans la gestion des ressources naturelles et bénéficient très peu des retombées de l'exploitation forestière, elles ne possèdent pas de FDL (Goma, 2020).

Malgré la présence d'un FDL doté d'une expérience d'une décennie, sa contribution réelle au développement local reste mitigée. La lenteur administrative dans la gestion opérationnelle de la SDC, le retard dans la livraison des matériels et intrants par le comité d'achat (décalage avec le calendrier agricole), la faible compétence des animateurs sur les outils de conception et de pilotage des projets, la faible prise en compte des préoccupations des groupes défavorisés (autochtones, femmes, etc.), la faible adhésion des CLPA aux microprojets communautaires sont parmi les problèmes organisationnels des investissements du FDL dans la mise en œuvre des microprojets. De plus, le protocole d'accord relatif à l'exploitation de bois de valeur commerciale dans la SDC de l'UFA Pokola entre la CIBOlam et le Conseil de concertation n'a pas été respecté totalement. Le protocole d'accord conclu exige le paiement de $2000 \mathrm{FCFA} / \mathrm{m}^{3}$ de bois exploité par la CIB pour les essences de catégorie 1 (Doussié et Wengué). Cependant lors du paiement de la taxe, le Wengué a été évalué et payé à $1000 \mathrm{FCFA} / \mathrm{m}^{3}$ au lieu de $2000 \mathrm{FCFA} / \mathrm{m}^{3}$, ce qui suppose un manque à gagner pour le FDL.

\section{CONCLUSION}

Le but de l'étude était d'évaluer le fonctionnement du FDL de la Série de Développement Communautaire (SDC) de l'UFA Pokola. Les deux objectifs spécifiques poursuivis étaient de décrire l'organisation et le fonctionnement du FDL, et analyser les ressources financières mobilisées et investit par ce fonds au profit des communautés locales et populations autochtones. Il ressort de l'étude que les trois principaux organes de gestion de la SDC sont le conseil de concertation, la coordination technique et le comité d'évaluation. Dans ces organes de gestion, les CLPA sont des parties prenantes au même titre que les représentants des acteurs institutionnels (conseil départemental de la Sangha, service déconcentré de l'état, la société forestière CIB-Olam, etc.). Le financement de la SDC est assuré le 
FDL, géré directement par le conseil de concertation. Ce fonds est créé dans le but de lutter contre la pauvreté et de contribuer à l'amélioration des conditions de vie des populations vivant dans la SDC de l'UFA Pokola. Il est alimenté principalement par les redevances issues de l'exploitation des essences forestières dans les unités forestières de production $\left(200 \mathrm{FCFA} / \mathrm{m}^{3}\right.$ de bois commercialisable exploité dans l'UFA) et dans la SDC (1000 à $2000 \mathrm{FCFA} / \mathrm{m}^{3}$ de bois exploité). Le FDL est bien connu des communautés locales et populations autochtones de la SDC de l'UFA Pokola (81\%). Pendant une décennie de fonctionnement, un montant de 182214333 FCFA a été mobilisé dont 145274472 FCFA a permis de financer près de 69 microprojets aux profits des CLPA de l'ensemble des localités de la SDC de l'UFA Pokola. L'investissement dans les microprojets de production végétale représente $64 \%$ de l'ensemble des investissements du FDL, avec une prédominance pour les cultures de cacaoyer et du manioc.Des insuffisances d'ordre institutionnel, organisationnel et les capacités techniques des acteurs sont à corriger afin d'optimiser l'utilisation des fonds disponibles et augmenter l'impact socioéconomiques de ces investissements.

\section{REMERCIEMENTS}

Les auteurs de l'article remercient les autorités administratives de la CIB-Olam ainsi que les membres de la cellule d'aménagement. Ils remercient également la Direction Départementale de l'Economie Forestière de la Sangha ainsi que toutes personnes qui ont contribué à l'amélioration de ce document.

\section{REFERENCES BIBLIOGRAPHIQUES}

[1] Anonyme, 1999. Principes et pratique de cogestion forestière : témoignages d'Afrique de l'ouest. Document $\mathrm{n}^{\circ} 2$ de l'Union Européenne sur la foresterie tropicale. OverseasDevelopment Institut Londres, Commission européenne, Bruxelles, $37 \mathrm{p}$.

[2] Anonyme, 2007. Plan d'aménagement de l'Unité Forestière d'Aménagement (UFA) de Pokola (2007-2035). Congolaise Industrielle des Bois (CIB), Terea, GTZ, FFEM, Groupe Agence Française de Développement, OIBT, 382 p.

[3] Ansallem I., 2015. Conventions internationales formelles. In : Mémento du Forestier Tropical. Editions Quae, pp. 110130.

[4] Arrêté $\mathrm{n}^{\circ} 2668$ du 15 avril 2010 portant institution, organisation et fonctionnement du Conseil de Concertation de la Série de Développement Communautaire de Pokola. Journal Officiel de la République du Congo, 16 : 343-345.

[5] Boutefeu B. 2005. La demande sociale de nature en ville : enquête auprès des habitants de l'agglomération lyonnaise. Lyon, Edition Puca-Certu, collection Recherches ${ }^{\circ} 154,81$ p.
[6] Collas de Chatelperron P., 2000. Fondements de la démarche du projet en matière de classement des forêts. In gestion durable des forêts au Cameroun : vers une foresterie responsable, contributions du projet forêts et terroirs. Actes de l'atelier d'échanges 4-6 juillet 2000, Yaoundé, CIRAD, $78 \mathrm{p}$.

[7] FAO, 1996. Critères et indicateurs pour l'aménagement des forêts de l'Afrique tropicale sèche. FAO, Rome, Italie, 35 p.

[8] FapaNanfack R., Gélinas N., Bobo Kandiri S., Asselin H., HiolHiol F., Tatsoula A., 2020. Détermination de la gouvernance forestière dans les forêts communautaires de 1'Est Cameroun. Bois et forêts des Tropiques, 343, pp. 5366.

[9] Fomété T., 2001. La fiscalité forestière et l'implication des communautés locales à la gestion forestière au Cameroun. Document du Réseau de foresterie pour le développement rural, pp : 18-29.

[10] Goma A.R., 2020. Analyse de l'implication des communautés locales et populations autochtones dans la gestion de l'Unité Forestière d'Exploitation Louvakou. Mémoire de Master en Sciences et Techniques Forestières, Ecole Nationale Supérieure d'Agronomie et de Foresterie, Université Marien Ngouabi, $67 \mathrm{p}$.

[11] Koffi Kouamékan J. M., 2013. Gestion participative, capabilités et résilience dans les forêts classées de Côte d'Ivoire.Ethics and Economics, 10(1): 119-152.

[12] MankeriNdobolo L.N., 2020. Etat de mise en ouvre du plan de gestion de la série de développement communautaire de l'UFA Kabo. Mémoire de Master en Sciences et Techniques Forestières, Ecole Nationale Supérieure d'Agronomie et de Foresterie, Université Marien Ngouabi, $43 \mathrm{p}$.

[13] N'zala D., 2002. Conservation et gestion durable des écosystèmes des forêts tropicales humides de l'Afrique Centrale. Exemple des concessions de Pokola-KaboLoundoungou, Congo. Etude FAO, 21 p.

[14] Nguinguiri J. C., 1999. Les approches participatives dans la gestion des écosystèmes forestiers d'Afrique centrale. Revue des initiatives existantes. Occasionalpaper $\mathrm{n}^{\circ} 23$, CIFOR (Center For International ForestryResearch), 24 p.

[15] Nkéoua G. et NgoyaKessi A. M., 2005. L'aménagement des forêts au Congo.URL : http://www.c3ed.uvsq.fr/cdg.ecorev/fr/pdf/t4/NkéouaNgoya.pdf.Consulté le 10 avril 2021.

[16] Robbin, Mcsweeney, Waite, Rice, 2006. Even Conservation Rules Are Made to Be Broken: Implications for Biodiversity. Environmental Management, 37 (2): 162169.

[17] Séne A., 2014. Implication des acteurs non étatiques dans la gouvernance des ressources naturelles au Sénégal : cas des ressources forestières à Zinguinchor et halieutiques à Mbour. Dans Kobor, D. (Ed.) Energie renouvelables et développement durable. Revue science Lib, Toulouse, Editions Mersenne, pp : 109-130. 